\title{
Improving Eyewitness Identifications From Lineups: Simultaneous Versus Sequential Lineup Presentation
}

\author{
R. C. L. Lindsay \\ Queen's University \\ Kingston, Ontario, Canada
}

\author{
Gary L. Wells \\ University of Alberta \\ Edmonton, Alberta, Canada
}

\begin{abstract}
Staged crime research has demonstrated the utility of controlling the conduct of lineups as a means of reducing false identifications with little or no apparent decline in the rate of correct identifications by eyewitnesses (e.g., Lindsay \& Wells, 1980; Malpass \& Devine, 1981 a; Wells, 1984). A recent variation in lineup procedure shows that a blank lineup, which includes no suspects, can reduce the rate of false identifications if it precedes the actual lineup. However, there are several practical problems that make it unlikely that police will accept this procedure. Sequential lineup presentation is proposed as a means of accomplishing the same goals of reducing false identifications with little or no loss in accurate identifications. A crime was staged for 240 unsuspecting eyewitnesses either individually or in pairs. One quarter of the eyewitnesses attempted identifications in each of four lineup conditions: Six pictures were presented either simultaneously, as used in traditional procedures, or sequentially, in which yes/no judgments were made for each picture; each procedure either contained the photograph of the criminal-confederate or a picture of a similar looking replacement. Sequential lineup presentation significantly reduced false identifications but did not significantly influence correct identifications when compared with the simultaneous procedure. This resulted in an overall increase in diagnosticity ratio (Wells \& Lindsay, 1980) using the sequential procedure. The data are interpreted as supporting the conclusion that sequential presentation of lineups can reduce false identifications of innocent suspects by reducing eyewitnesses' reliance on relativejudgment processes.
\end{abstract}

Wells (1978) argued that two types of variables could be distinguished in eyewitness research: (a) estimator variables, such as race or sex, which may affect eyewitness accuracy but are not controllable in actual cases and (b) system variables, such as lineup structure, which not only affect accuracy but also can be controlled. The potential value of researching system variables is as a means of improving on current procedures. To date, system-variable research has been conducted on questioning procedures, and the resultant accuracy of eyewitness memory has been reflected in descriptions of people and events

This research was supported by a grant from the Social Sciences and Humanities Research Council of Canada to the first author.

Requests for reprints may be sent to R. C. L. Lindsay, Department of Psychology, Queen's University, Kingston, Ontario, Canada K7L 3N6 or Gary L. Wells, Department of Psychology, University of Alberta, Edmonton, Alberta, Canada T6G 2E9.
(Loftus, 1979); however, relatively little stagedcrime, system-variable research has dealt with issues of eyewitness identification.

Investigation of eyewitness identification accuracy has demonstrated considerable variance in the rate of correct and false identifications. In particular, false identifications of innocent people have been obtained from as few as $12 \%$ (Leippe, Wells, \& Ostrom, 1978) or as many as $78 \%$ (Malpass \& Devine, 1981a) of the subjects in some conditions of staged crime experiments. If this variance in rate of false identification is produced by differences in system variables, substantial reductions in the risk of real world identification errors might be obtained by following appropriate procedures.

Lineup and photo-spread identification procedures typically involve the presentation to the eyewitness(es) of one suspect embedded among "foils" or "distractors." A foil or distractor is a stand-in who is not a suspect 
but instead is known to be innocent (e.g., police officers or persons drawn from jail cells). Although some lineups may be composed entirely of suspects, the opinions of legal experts (e.g., Sobel, 1973) and psychologists (e.g., Ellison \& Buckhout, 1981; Wells \& Lindsay, 1980) clearly argue against such practices. The advantages of having knowninnocent foils in lineups are numerous, and single-suspect lineups are therefore the stateof-the-art model on which the current study is based. Lineups may be live (the so-called corporeal lineup) or color photographs. Increasingly, police departments are using the photographic method for several reasons, including the greater ease with which acceptable distractors can be found and the fact that right to counsel does not accrue to the suspect in the case of photographic identification procedures. Although the latter reason seems especially questionable (see Grano, 1984), it should be noted that there is no evidence that photo identifications are less reliable than live identifications, even when the witnessed event was a live event (Shepherd, Ellis, \& Davies, 1982).

Lindsay and Wells (1980) outlined the possible outcomes that can result from a lineup identification procedure using the single-suspect model. If the lineup contains the perpetrator, the eyewitness can make an accurate identification, a foil identification, or an incorrect nonidentification. If the lineup does not contain the perpetrator, the eyewitness can make a false identification, a foil identification, or a correct nonidentification. In the real world case, only foil identifications are known at the time to be eyewitness errors. (Indeed, this is one of the reasons why it is important in actual cases to have foils rather than all suspects-so that at least one type of error can be detected with certainty.) Identifications of the suspect and nonidentifications may be either accurate or inaccurate depending on whether the lineup contains the actual perpetrator.

It is important to keep in mind that the identification of a foil is a known error and does not function as a false identification in the true sense-for example, charges will not be brought against Officer Jones if he is identified. Thus, when using the single-suspect lineup model, false identifications can occur only in perpetrator-absent lineups. This important observation has paved the way for Bayesian analyses of lineups (Wells \& Lindsay, 1980). Using this Bayesian approach, Wells and Lindsay outlined the two main statistical properties of lineup identifications, nonidentifications, and foil identifications; namely diagnosticity and informativeness. Diagnosticity is a directional likelihood ratio, whereas informativeness is a combination of the likelihood ratio with prior probabilities. The simpler of the two, diagnosticity, captures the important elements of the lineup procedure because higher levels of diagnosticity produce greater informativeness for all prior probabilities greater than zero $(0)$ and less than one (1.0). The diagnosticity of an identification procedure is defined as the ratio of accurate identifications to false identifications. As indicated earlier, accurate identifications occur only in perpetrator-present lineups, and false identifications occur only in perpetratorabsent lineups. Similarly, the diagnosticity of foil identifications is the ratio of foil identifications in perpetrator-absent lineups to foil identifications in perpetrator-present lineups. The diagnosticity of nonidentifications is the ratio of nonidentifications in perpetrator-absent lineups to nonidentifications in perpetrator-present lineups. Obviously, the higher the diagnosticity ratio the more informative or diagnostic the identification decision from the lineup will be with regard to the guilt or innocence of the suspect. Higher diagnosticity ratios for identifications can result from increases in accurate identifications, decreases in false identifications, or both. These may result from better witnesses or better testing procedures. The current study randomly assigned witnesses to testing procedures so that diagnosticity differences are attributable to testing procedures.

There are three previous studies that have systematically varied eyewitness-identification testing procedures in attempting to find improvements in diagnosticity. ${ }^{1}$ Malpass and

\footnotetext{
' Another study (Malpass \& Devine, 1981b) attempted to vary testing procedures with lineups, but its status is not yet clear because of the lack of a perpetrator-absent lineup control.
} 
Devine (1981a) staged a vandalism and had eyewitnesses attempt an identification under conditions in which the perpetrator was present in or absent from the lineup and eyewitnesses were led to think the vandal was present or "may or may not" be present. Their results were a clear demonstration of the importance of telling eyewitnesses that the true perpetrator may not be in the lineup. Their results showed a robust decline in the likelihood of false identifications (from $78 \%$ down to $33 \%$ ) and no loss in accurate identifications $(75 \%$ to $83 \%)$ for the "is present" versus "may or may not be present" conditions, respectively. Their results translate into diagnosticity ratios of .96 and 2.52 , respectively, for the two sets of instructions. This is a profound demonstration of the potential for meaningful improvements resulting from system variable research with lineups.

Lindsay and Wells (1980) demonstrated that further improvement can be obtained even under conditions in which eyewitnesses are told that the perpetrator may or may not be present. Specifically, they varied the physical similarity of lineup members and found that lineups containing consistently poor foils (i.e., foils that fail to match the general description of the perpetrator) produce lower levels of diagnosticity than do lineups containing distractors that tend to match the general description of the perpetrator. It is important to note for later purposes that the similarity variable had no effect on willingness of eyewitnesses to attempt an identification. Instead, high similarity resulted in a spread of choices from the innocent suspect to known-innocent foils (in perpetrator-absent lineups) without comparable spread from the guilty suspect to foils (in the perpetratorpresent lineup).

Recently, Wells (1984) proposed that the improvements in diagnosticity resulting from instructions (Malpass \& Devine, 1981a) and similarity (Lindsay \& Wells, 1980) could be understood to result from a tendency for eyewitnesses to make "relative judgments." That is, eyewitnesses tend to choose the lineup member who most looks like the perpetrator relative to the other lineup members (cf. a comparison of each lineup member to one's recollection on a more "absolute" basis of comparison). Clearly, the relative-judgment strategy is adequate when the perpetrator is present in the lineup. However, when the perpetrator is absent, the relative-judgment strategy necessarily elicits errors. Telling eyewitnesses that the perpetrator may not be in the lineup helps reduce false identifications because it makes apparent the fallacious use of the relative-judgment strategy under perpetrator-absent conditions.

The way in which the similarity variable affects diagnosticity may also be understood in terms of the relative-judgment conceptualization. The pattern of data in the Lindsay and Wells (1980) study shows that increases in similarity between foils and suspect have little or no effect on witnesses' ability to choose the suspect when the suspect is guilty (i.e., he remains the best choice relative to other lineup members) but serves to spread choices to foils in the perpetrator-absent lineups (i.e., where the suspect is innocent).

Based on the relative-judgment conceptualization, Wells (1984) proposed that those eyewitnesses most prone to making relative judgments could be "screened" or "lured" by a blank lineup. A blank lineup is one containing no suspect (i.e., composed entirely of foils who are known to be innocent). He exposed unsuspecting students to a staged crime and subsequently asked them to attempt to identify the culprit. Half of the 192 eyewitnesses participated in the standard identification procedures, with 48 viewing a lineup containing a photo of the criminal and 48 a lineup in which the guilty party was replaced by a similar other. The remaining 96 participants first viewed a "blank" lineup containing no suspect and then were shown one of the lineups seen by other witnesses. Eyewitnesses who made a selection from the blank lineup (those that Wells's procedure would screen out) were significantly less likely to accurately identify the guilty party, significantly less likely to make an accurate no-identification decision, and significantly more likely to identify another innocent person from the second lineup than eyewitnesses who made a no-identification decision from the blank lineup. Compared to the nonscreened control group, eyewitnesses who successfully passed the screening test 
(i.e., those not making an identification from the blank lineup) made significantly fewer false identifications and significantly more correct no-identification decisions in the absence of the criminal as well as significantly fewer incorrect identifications (choices of foils) in the presence of the criminal.

Clearly the screening of eyewitnesses that tend to employ a relative-judgment strategy successfully increased the accuracy of resultant identification decisions. However, some practical limitations might reduce police willingness to adopt this technique. Among their objections would be the requirement of conducting two lineups, thus recruiting twice as many foils. All eyewitnesses making a selection from the first lineup would be discarded-not a problem for a researcher who simply runs another student through a staged crime but a potentially serious loss in a realworld case involving a single eyewitness. Furthermore, the technique may cease to be of value if the public became aware of the fact that two lineups were commonly used and that the "real" suspect appears in the second (a technique recently portrayed in Hill Street Blues).

What is needed is a procedure that reduces the tendency to employ a relative-judgment process without (a) eliminating eyewitnesses from the "real" identification test, (b) increasing the cost and workload for the police (i.e., by increasing the number of people required to conduct a lineup), or (c) allowing potential eyewitnesses to predict when or where the true suspect is likely to appear in the procedure. The absolute number of people required could be reduced by limiting the "arrays" to less than six people each. Similarly, the public could be prevented from anticipating the appearance of the suspect by randomly assigning the suspect to an array while informing the witness that more than one array will be employed. The knowledge that more than one array or lineup will be presented ought to reduce eyewitnesses' reliance on relative judgments because the witness has no means of determining in which array the suspect will appear. If this reasoning is correct, the only remaining issues to be determined are the optimal number of targets per array and total number of arrays.
Because an ideal procedure would avoid police objections, the total number of people involved in the identification procedure could be limited to the number typically employed in a single lineup. Assuming a six-person lineup, for example, the proposed technique could employ two sets of three, three sets of two, or six individuals presented sequentially. Because the idea is to prevent as much as possible the opportunity for eyewitnesses to make relative judgments, we opted for the sequential technique, in which the eyewitness sees one lineup member at a time, makes a yes/no decision for each lineup member on first viewing, and is not informed of the total number to be viewed. If the relative-judgment strategy is a major source of false identifications, the sequential procedure ought to be a powerful means of preventing relative judgments; it should force eyewitnesses to compare each lineup member to their recollection of the perpetrator using some absolute standard of recognition rather than considering who "most" looks like the perpetrator.

\section{Method}

\section{Participants}

Introductory psychology students were recruited by telephone to participate in a study of "complex information processing." A total of 243 Queen's University students volunteered to participate. From 1 to 4 students took part in each session.

\section{Procedure}

The experimenter met the participants at the laboratory door, led them into a large room $(9 \mathrm{~m} \times 10 \mathrm{~m})$, and then led them into a small cubicle $(3 \mathrm{~m} \times 3 \mathrm{~m})$ containing a table and two chairs (if more than two students were available for a session, a second similar cubicle off the same large room was employed). The cubicle(s) had a counter at waist level on two walls with drawers and cupboards below the counter and cupboards above the counter. After seating the participants, the experimenter explained that she had to leave briefly to "get some forms" and closed the door to the cubicle on her way out. Approximately $30 \mathrm{~s}$ after the experimenter left, a 21-year-old male Caucasian entered the cubicle. Looking directly at the subjects he stated that he "didn't know anyone was in this room" and would be "out of the way in just a minute." He then searched through several drawers and cupboards along both walls finally finding a calculator in a leather case. With the calculator in hand, he crossed to the door while looking in the direction of the (now) eyewitnesses and left, closing the door behind him. The entire event lasted approximately $20 \mathrm{~s}$. 
The experimenter returned shortly after the man had left and explained that the thief was a confederate and that the students had witnessed a staged crime. All participants received an explanation of the true purpose of the experiment (to investigate eyewitness identification accuracy) and were asked to sign a consent form. ${ }^{2}$ Witnesses were then separated (if more than one witness was in the cubicle) and completed an open-ended description of the criminal, then a detailed questionnaire about his appearance. Approximately $5 \mathrm{~min}$ after viewing the crime, the eyewitnesses were asked to attempt to identify the criminal from photographs.

\section{Identification Procedures}

Half of the eyewitnesses (simultaneous presentation conditions) were shown a board on which was mounted a set of six photographs of male Caucasians in their early 20s. Each picture was a $7 \mathrm{~cm}$ by $7 \mathrm{~cm}$, head and shoulder view from the front. For 60 of these witnesses the array contained a photograph of the criminal confederate, whereas for the remaining 60 , his picture was replaced with a photograph of a man who resembled him. The replacement photograph was selected on the basis of pilot testing to be the most similar in appearance to the confederate of 18 available photos of men fitting the general description. Although some studies and realworld cases have demonstrated that innocent suspects may be identified even when they are not particularly similar in appearance to the criminal, a stronger test of the value of sequential lineup presentation was provided by having a similar-looking innocent suspect. The photographs were numbered from 1 to 6 and were presented in two rows of three photographs. Each witness was also provided with an identification form. Across the middle of the form were six boxes containing the numbers 1 through 6. Below there was a seventh box labelled "none of them." The instructions asked the witness to put an "X" through the appropriate box. At the bottom of the form the witnesses were asked to rate on a 7-point scale how certain they were that their identification decision was correct. The experimenter placed the picture array, covered by the identification for $m$, on the table in front of the eyewitness. Before the eyewitness looked at the photographs, the experimenter pointed to and read aloud the following statement from the top of the identification form: "Remember, as in a real identification situation, the guilty party may or may not be present." After reading this statement the experimenter immediately left the room and returned $3 \mathrm{~min}$ to $5 \mathrm{~min}$ later. All witnesses completed the identification form in the alloted time.

Witnesses in the sequential lineup presentation conditions were provided with a different identification form. The identically worded warning that the criminal may or may not be in the lineup was at the top of the form. The next line read "Is \#1 the person you saw? No, Yes" and was followed by a 7-point certainty scale. Eleven more lines followed, identical in every regard except that the number varied from 2 through 12 . The experimenter explained that the eyewitness would be shown a sequence of individual photographs and must decide for each whether or not it was a picture of the criminal. It was emphasized that the witness could take as long as he or she wished to decide but that each photograph would only be seen once. The experimenter held in her hand a "deck" of 12 photographs, face down. Thus the experimenter first read the warning, then asked "Is number one the person you saw?" then turned a photograph over in front of the witness and waited for the witness to circle either no or yes and a second number indicating how certain the witness was of this decision. After both responses were circled, the photograph was placed on the bottom of the deck and the procedure repeated. Following the sixth photograph the experimenter explained that there would be no further pictures. Witnesses were intentionally led to believe that there might be more than six photographs to reduce any tendency to increase the probability of making a "yes" response as the end of the pile approached.

Half of the sequential lineup eyewitnesses were shown six photos including a picture of the confederate-thief. The remaining half saw a photo of the innocent suspect rather than the guilty party. The same five photographs of foils were used in all conditions. Twenty eyewitnesses from each of the four experimental conditions (simultaneous vs. sequential presentation by perpetrator present vs. absent) saw the suspect's photograph in the first, third, or fifth position. The order of foils in Positions 2, 4 and 6 was constant, and the position of all foils was constant for a given position of the suspect, regardless of mode of presentation. For the sequential presentation conditions, the confederate arranged the order of the deck in advance, and thus the experimenter was blind to the position of the suspect's picture at least until it was turned over. She was instructed to ignore the pictures and to restrict her gaze to the witnesses' identification forms to the best of her ability.

The presence of the experimenter in the room during the identification procedure is confounded with mode of presentation. However, absence of the experimenter for the simultaneous procedure is consistent with attempts to minimize experimenter or police officer effects (as recommended by Brooks, 1983). Keeping the experimenter blind to position and asking her to look only at the response sheet rather than the photographs was intended to reduce or prevent such effects in the sequential presentation conditions.

After the identification procedure was completed, all witnesses who had identified the suspect (guilty or innocent) were asked if they would be willing to testify in a mock trial later in the year. The purpose of this measure was not only to recruit eyewitnesses for a subsequent

\footnotetext{
${ }^{2}$ Three subjects refused to sign the consent form. Upon questioning they reported that the signing of a "legal document" for such a mundane procedure seemed like "overkill" and they suspected that the form was deceptive (it was not). These subjects did not participate further. In spite of this response from these three people, recent research suggests that debriefing subject-witnesses at this point has no appreciable effect on the results (Murray \& Wells, 1982). In other words, it seems that incidental encoding is the primary prerequisite for creating an analog to the actual eyewitness situation and that continuing the ruse beyond this point is unnecessary.
} 
mock trial study but also to test the differential-attrition hypothesis (Wells, Lindsay, \& Ferguson 1979), which states that inaccurate eyewitnesses may be less willing to testify in court than accurate eyewitnesses. All students were debriefed and thanked for their assistance.

\section{Results}

\section{Lineup Position}

For sequential lineup presentation to be a viable alternative, it is important that the results of the procedure not be unduly influenced by order effects (i.e., the position of the suspect). There were no significant main effects nor interactions involving the suspects' position in the lineup on the rate of identification of the perpetrator, innocent suspect, or foils; nor were there significant main effects or interactions on the rate of no-identification decisions, $\chi^{2}<1$ for each of these variables. Position of the suspect had no significant effects on confidence of decision regardless of the decision made ( $F<1$ in all cases).

\section{Identification Decision}

The primary hypothesis of this experiment was that sequential lineup presentation would inhibit the use of relative judgments and result in fewer false identifications and more no-identification decisions. The proportions of identifications of the suspect, foils, and no-identification decisions under each lineup condition are presented in Table 1. As expected the suspect was significantly more likely to be identified when guilty than innocent $\left(.55\right.$ vs. .30 , respectively, $\chi^{2}=8.80$, $p<.005$ ). The suspect was also significantly more likely to be identified using the simultaneous as compared to the sequential lineup procedure $\left(.51\right.$ vs. .34 , respectively, $\chi^{2}=3.92$, $p<.05$ ). However, the higher rate of suspect identification with simultaneous presentation was primarily due to a higher rate of false identifications using this procedure. The two procedures did not result in significantly different rates of correct identification, $\chi^{2}(1$, $N=240$ ) $<1$, $n s$, but did differ significantly in false identification rates, $\chi^{2}(1, N=$ $240)=7.11, p<.01$. As a result, simultaneous lineup presentation produced a much lower diagnosticity ratio than did sequential lineup presentation ( 1.35 vs. 3.06 , respec-
Table 1

Proportion of Eyewitnesses Identifying the Suspect, Identifying a Foil, or Making No Identification From Lineups

\begin{tabular}{lccccc}
\hline & \multicolumn{3}{c}{$\begin{array}{c}\text { Perpetrator's status and } \\
\text { mode of lineup presentation }\end{array}$} \\
\cline { 2 - 3 } & \multicolumn{2}{c}{$\begin{array}{c}\text { Perpetrator } \\
\text { present }\end{array}$} & & \multicolumn{2}{c}{$\begin{array}{c}\text { Perpetrator } \\
\text { absent }\end{array}$} \\
\cline { 2 - 3 } $\begin{array}{c}\text { Identification } \\
\text { decision }\end{array}$ & $\begin{array}{c}\text { Simul- } \\
\text { taneous }\end{array}$ & $\begin{array}{c}\text { Sequen- } \\
\text { tial }\end{array}$ & $\begin{array}{c}\text { Simul- } \\
\text { taneous }\end{array}$ & $\begin{array}{c}\text { Sequen- } \\
\text { tial }\end{array}$ \\
\hline $\begin{array}{c}\text { Identified } \\
\text { suspect }\end{array}$ & .58 & .50 & .43 & .17 \\
$\begin{array}{c}\text { Identified } \\
\text { foil }\end{array}$ & .12 & .02 & .15 & .18 \\
$\begin{array}{c}\text { No identifi- } \\
\text { cation }\end{array}$ & .30 & .48 & .42 & .65 \\
\hline
\end{tabular}

tively). The significance of this difference can be tested by evaluating the interaction of lineup presentation with presence versus absence of the criminal from the lineup (Langer \& Abelson, 1972). This interaction was significant $(z=2.76, p<.005)$. The total number of incorrect identifications of innocent people (innocent suspects and foils) may also be of interest because police do not always restrict lineups to a single suspect. Overall, $35.0 \%$ of eyewitnesses exposed to a simultaneous lineup identified an innocent person, whereas only $18.3 \%$ of eyewitnesses shown the same lineups sequentially identified innocent people, $\chi^{2}(1, N=240)=6.25$, $p<.025$.

The sequential presentation of photographs presented the possibility that eyewitnesses might identify more than one person from the lineup. However, only three witnesses $(2.5 \%)$ did so. One first identified a foil then the guilty suspect. The remaining two, both in the criminal-absent conditions, each identified two foils. (All three were treated as foil identifications in the data analysis because each identified at least one person known to be innocent.) A lower rate of no-identification decisions was obtained with simultaneous as compared to sequential lineup presentation (.36 vs. .56$), \chi^{2}(1, N=240)=5.24, p<.025$. The rate of no-identification decisions did not differ significantly across perpetratorpresent and perpetrator-absent conditions (.40 
vs. .54 , respectively), $\chi^{2}(1, N=240)=2.93$, $p<.10$, nor was there a significant interaction $(z=0.15)$.

\section{Volunteering to Testify}

The proportion of eyewitnesses who identified the suspect and volunteered to testify was used as a test of the differential attrition hypothesis. Contrary to the hypothesis, inaccurate eyewitnesses were significantly more likely than accurate eyewitnesses to volunteer to appear in court (.86 vs. .67 , respectively), $\chi^{2}(1, N=102)=9.51, p<.01$. The rate of volunteering was particularly low for accurate eyewitnesses who had viewed the lineup sequentially. As a result, there was a significantly lower overall rate of volunteering from the sequential as compared to the simultaneous lineup presentation conditions (.66 vs. .80 , respectively), $\chi^{2}(1, N=102)=4.37, p<.05$. The interaction was significant as well $(z=$ $2.18, p<.02$ ). Simple effects analyses revealed that accurate as compared to inaccurate eyewitnesses were nonsignificantly less likely to testify when they had viewed the simultaneous lineups, $\chi^{2}(1, N=62)=1.33$, $n s$, but significantly less likely to testify if they had viewed sequential lineups, $\chi^{2}(1, N=40)=12.10$, $p<.01$.

\section{Confidence and Accuracy}

As is typical in this research, eyewitnesses who identified the guilty party were slightly more confident than those who identified the innocent suspect, resulting in a small but significant confidence-accuracy correlation $(r=.30, n=102, p<.001)$. Similarly, eyewitnesses making a correct no-identification decision were slightly more confident than those making an incorrect no-identification decision, again resulting in a significant confidence-accuracy correlation $(r=.19, n=$ $110, p<.025)$. Mode of lineup presentation did not significantly influence eyewitness confidence, nor did it interact with other variables to influence confidence. Overall identifications of the suspects and no-identification decisions were made with equal confidence ( $M=4.93$ vs. 4.95 , respectively, $F>$ 1). As reported elsewhere (Lindsay, 1985), identifications of foils were made with significantly lower confidence than other identification decisions $(M=4.18$ vs. 4.94 , respectively, $F=6.20, p<.01$ ).

\section{Discussion}

The data provide support for the idea that a sequential lineup procedure yields greater diagnosticity ratios than does the common simultaneous lineup procedure. This increase in diagnosticity resulted from a robust reduction in the rate of false identifications in the perpetrator-absent conditions in conjunction with no change in the rate of accurate identifications. The reduction of inaccurate identifications without loss of accurate identifications holds true regardless of whether the single-suspect model or multiple-suspect model is used. This pattern of results is consistent with the improvements found to result from wording variations (Malpass \& Devine, 1981a), physical similarity variations (Lindsay \& Wells, 1980), and the use of blank lineups (Wells, 1984). That is, improvements seem to result almost exclusively from reductions in inaccurate identifications; none of these techniques has shown improvements in the rate of accurate identifications. We suggest that each of these system-variable improvements can be understood within the relativejudgment conceptualization. Specifically, we propose that (a) instructional wordings that warn that the perpetrator may not be present tend to make salient to witnesses the potential danger of relative judgments; (b) increased similarity between suspect and foils tends to spread relative judgment errors to foils, but only if the perpetrator does not appear in the lineup; (c) blank lineups tend to screen out those most prone to making relative judgments; and (d) sequential presentations, which require decisions on each lineup member at first presentation, force witnesses away from the possibility of using a relative-judgment strategy.

The consistency of the data from four experiments employing different confederates and lineups conducted at three widely dispersed universities with delays between event and identification varying from a few minutes to three days reduces the probability that the 
results are an artifact of any particular procedure, and strengthens our belief that all of these effects are mediated by relative judgment processes. However, the reader is cautioned that very little staged-crime, system-variable research exists, and the strength of our conclusions would be enhanced considerably by replications of the reported effects by independent laboratories. In particular, interactions of multiple variables in the same study have not been explored.

Although the sequential procedure improved diagnosticity, it had the unexpected negative effect of decreasing the willingness of accurate eyewitnesses to testify in court. If this differential willingness to testify were to generalize to actual court cases, approximately half the gain in diagnosticity attributable to the sequential procedure would be lost. However, we propose two reasons why we would not expect such differential willingness to influence actual cases. First, realworld eyewitnesses would likely experience implicit pressure (e.g., civil duty) and explicit pressure (police statements about the importance of the eyewitness to their case) to testify. As well, the eyewitness can be subpoenaed. Regardless of how they are brought to court, they are likely to be equally convincing because the confidence measure showed that sequential-procedure eyewitnesses were no less confident than were simultaneous-procedure eyewitnesses. (Previous research indicates that confidence is the major determinant of belief, c.f. Lindsay, Wells, \& Rumpel, 1981.)

The results indicate that the sequentiallineup procedure has beneficial effects on diagnosticity above and beyond merely cautioning witnesses that the perpetrator may not be in the lineup. As well, the sequential procedure has certain advantages over the mere assurance of reasonable physical similarity between lineup members. Specifically, increases in physical similarity help protect the innocent suspect by spreading eyewitnesses' choices to foils. This means that the similarity variable is not generalizable to lineups in which all members are suspects.

Sequential lineup presentation differs from both mug shot and show-up procedures in important ways. Mug shots include only pic- tures of people previously convicted of crimes. All pictures are of potential suspects; thus any choice will be treated as a potentially valid identification (at least until further investigation is conducted). Sequential lineups, indeed all lineups, should contain a single suspect and no indication that any of the people in the lineup have criminal records. Show ups are highly suggestive because only a single face is shown; however, a witness shown a sequential lineup is aware that more faces are available and that the suspect may appear in any position in the sequence. As a result, sequential lineup presentation need not produce the biases associated with either mug shot or show-up procedures. The sequential procedure also has advantages over the blank-lineup control procedure. The use of blank lineups rests on the assumption that the public will remain ignorant of the fact that the first lineup does not contain a suspect. As well, blank lineups require more foils and therefore more effort and cost. Even more damaging is the fact that use of a blank lineup inevitably impeaches some eyewitnesses, a cost that may be too great in real world cases. It is the latter factor that will likely make police reluctant to consider the use of the blank-lineup procedure. The sequential lineup, however, appears to work for both the multiple-suspect and the single-suspect models, does not rest on the assumption that the public remain ignorant of how it works, does not require more foils, and does not make a deceptive implication that the suspect appears in any particular position among a set of persons.

\section{References}

Ellison, K. W., \& Buckhout, R. (1981). Psychology and criminal justice. New York: Harper \& Row.

Grano, J. D. (1984). Legal perspective on problems inherent in eyewitness identification. In G. L. Wells \& E. F. Loftus (Eds.), Eyewitness testimony: Psychological perspectives (pp. 315-335). New York: Cambridge University Press.

Langer, E. J., \& Abelson, R. P. (1972). The semantics of asking a favour. How to succeed in getting help without really dying. Journal of Personality and Social Psychology, 24, 26-32.

Leippe, M. R., Wells, G. L., \& Ostrom, T. M. (1978). Crime seriousness as a determinant of accuracy in eyewitness identification. Joumal of Applied Psychology, $63,345-351$. 
Lindsay, R. C. L. (1985). Confidence and accuracy of eyewitness identification from lineups. Manuscript under editorial review.

Lindsay, R. C. L., \& Wells, G. L. (1980). What price justice? Exploring the relationship of lineup fairness to identification accuracy. Law and Human Behavior, 4, 303-313.

Lindsay, R. C. L., Wells, G. L., \& Rumpel, C. M. (1981). Can people detect eyewitness-identification accuracy within and across situations? Journal of Applied Psychology, 66, 79-89.

Loftus, E. F. (1979). Eyewitness testimony. Cambridge, MA: Harvard University Press.

Malpass, R. S., \& Devine, P. G. (1981a). Eyewitness identification: Lineup instructions and the absence of the offender. Journal of Applied Psychology, 66, 482489.

Malpass, R. S., \& Devine, P. G. (1981b). Guided memory in eyewitness identification. Journal of Applied Psychology, 66, 343-350.

Murray, D. M., \& Wells, G. L. (1982). Does knowledge that a crime was staged affect eyewitness accuracy? Journal of Applied Social Psychology, 12, 42-53.

Shepherd, J., Ellis, H. D., \& Davies, G. M. (1982). Identification evidence: $A$ psychological evaluation. Aberdeen, Scotland: Aberdeen University Press.
Sobel, N. R. (1973). Eyewitness identification: Legal and practical problems. New York: Clark Boardman.

Wells, G. L. (1978). Applied eyewitness-testimony research: System variables and estimator variables. Journal of Personality and Social Psychology, 36, 15461557.

Wells, G. L. (1984). The psychology of lineup identifications. Journal of Applied Social Psychology, 14, (2), 89-103.

Wells, G. L., \& Lindsay, R. C. L. (1980). On estimating the diagnosticity of eyewitness nonidentifications. Psychological Bulletin. 88, 776-784.

Wells, G. L., \& Lindsay, R. C. L. (1985). Methodological notes on the confidence-accuracy relationship in eyewitness identifications. Journal of Applied Psychology, $70,413-419$.

Wells, G. L., Lindsay, R. C. L., \& Ferguson, T. J. (1979). Accuracy, confidence, and juror perceptions in eyewitness identification. Journal of Applied Psychology, 64, 440-448.
Received October 15, 1984 Revision received January 7, 1985 